\title{
Creating Audio Products With Maximum End-User Value
}

\author{
WOLFGANG KLIPPEL, AES fellow \\ KLIPPEL GmbH, Mendelssohnallee 30, 01277 Dresden, Germany (wklippel@klippel.de)
}

\begin{abstract}
The value assigned by the end user to a loudspeaker, headphone, or any other audio device determines his purchase decision and the success of the product in the market. The paper investigates the relationship between end-user value, performance characteristics, cost structure, and the particular design. A model based on modified benefit-cost ratio is presented that describes the impact (sensitivity) of the performance characteristics on the end-user value. The performance sensitivity is a central and powerful term in audio engineering because it links physical, perceptual, and economical quantities. This new concept is applied to all phases of the product life and addresses open questions such as defining the optimum target performance, selecting design choices, increasing the yield rate in production, and ensuring reliability and quality in the final application.
\end{abstract}

\section{INTRODUCTION}

The success of a new audio product in the market depends on the value as seen by end users. The paper picks up this claim and raises questions of how to define and measure this value over the complete product life [1].

After defining the end-user value, performance, and design sensitivity in Secs. 2-4, the paper discusses the assessment of the performance over the complete life cycle as illustrated in Fig. 1. Sec. 5 investigates the target application where marketing analyzes the expectations of the end user considering the influence of the acoustical environment and selected stimulus. Listening tests and measurements of competitive products, experience, and intuition lead to a clear definition of the target performance, which is the basis for the product development. Sec. 6 discusses how standard measurements, numerical simulations, and other tools can be used to find the optimum design maximizing the end-user value. The role of fast and comprehensive testing at the end-of-line (EoL) for quality assurance and process control will be addressed in Sec. 7 in order to increase the yield rate in production. Finally, Sec. 8 presents new ways to cope with aging, fatigue, and field rejects, which reduce the customer satisfaction in the final application.

\section{END-USER VALUE}

This section introduces important terms and concepts that are required to investigate the end-user value of complete products but can also be applied to transducers and other audio components. An experienced audio engineer who is familiar with the conflict between engineering, human perception, and economy uses those ideas intuitively in his or her daily work. The mathematical apparatus complementing the explanations in this section gives a solid basis for the following sections but is not essential for the general understanding.

The term end-user value used in this paper is an economical category corresponding to the sales volume of a particular product summarizing the purchase decision of many customers.

Taking a black box model, the end-user value

$$
V=f_{\mathrm{VD}}(\mathbf{D})
$$

depends on design variables $\mathbf{D}=\left\{d_{1}, \ldots, d_{k}, \ldots\right\}$ describing the geometry and selected material (e.g., cone) and manufacturing process (e.g., assembling).

The multi-variate function $f_{\mathrm{VD}}$ is usually not known because it varies with particular application and time period. This function is also difficult to model because it is quite complex and even nonlinear due to perceptual and cognitive evaluation by the end user. A deep neural network can be used to describe this complicated relationship but requires blind machine learning of the free model parameters based on known design variables $\mathbf{D}$ and the corresponding sales volume as an indicator of the end-user value $V$. This approach needs a big data pool with a large number of products and sufficient design information. 


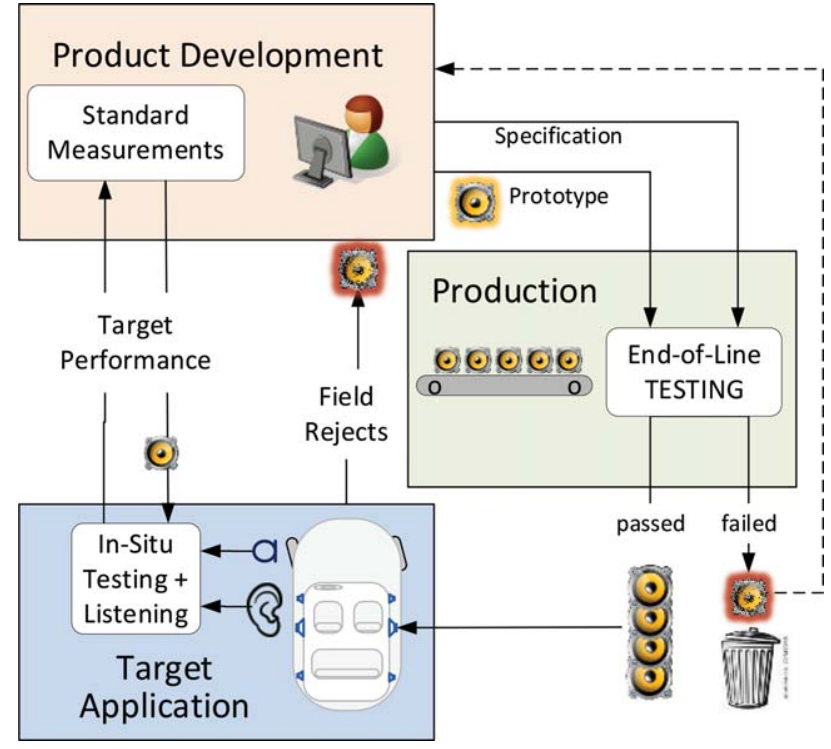

Fig. 1. Life cycle of a transducer used in automotive application.

This paper here focuses on the objective of finding the optimum product design $\mathbf{D}^{*}$ that maximizes the value $V$ :

$$
\mathbf{D}^{*}=\arg \max _{\mathbf{D}}\{V(\mathbf{D})\}
$$

This task can be solved by using an iterative search for the optimum design

$$
\mathbf{D}[i+1]=\mathbf{D}[i]+\Delta \mathbf{D}[i] i=1,2, \ldots
$$

starting with a known design D [1] of a similar product used in the same field of application and applying a design change $\Delta \mathbf{D}$ that generates a modified end-user value:

$$
V(\mathbf{D}+\Delta \mathbf{D})=V(\mathbf{D})+\Delta V(\Delta \mathbf{D})
$$

In order to minimize the number of iterations, the design change $\Delta \mathbf{D}$ should be chosen carefully to always generate a positive increment $\Delta V$. This objective can be accomplished by considering the sensitivity $s_{k}(\mathbf{D})$ of the design variable $d_{k}$ [2], which is a first-order partial derivative of Eq. (1) with respect to $d_{k}$ evaluated at the point defined by the current design $\mathbf{D}$. It can be estimated as the difference quotient

$$
s_{k}[i] \approx \frac{\Delta V}{\Delta d_{k}}=\left.\frac{V[i]-V[i-1]}{d_{k}[i]-d_{k}[i-1]}\right|_{\Delta d_{j}=0 \forall j \neq k}
$$

considering only a small change in one design variable $d_{k}$.

The change of $\Delta V$ can be described by a Taylor series truncated after the linear term

$$
\left.\Delta V \approx \sum_{\forall k} s_{k}(\mathbf{D}) \Delta d_{k}\right|_{\Delta \mathbf{D}<<\mathbf{D}}
$$

using the design sensitivity $s_{k}(\mathbf{D})$ as a weight scaling the design change $\Delta d_{k}$. This linearized model can be used to calculate $\Delta \mathbf{D}$ in Eq. (3), giving an iterative search for the optimum design values

$$
\begin{gathered}
d_{k}[i+1]=d_{k}[i]+\Delta d_{k}[i] \quad \forall k \\
=d_{k}[i]+\mu \frac{d_{k}[i]-d_{k}[1]}{s_{k}[i]-s_{k}[1]} s_{k}[i]
\end{gathered}
$$

using the design sensitivity $s_{k}[\mathrm{i}]$ as defined in Eq. (5) and a step size parameter $\mu<1$. In each iteration step the design sensitivity will be reduced and the end-user value increased. The design sensitivity $s_{k}\left(\mathbf{D}^{*}\right)$ of all variables becomes zero at local maxima of the end-user value, and the iteration is converged to the optimum values in design variables $\mathbf{D}^{*}$ according Eq. (2) if a global maximum is found.

\section{BENEFIT-COST RATIO}

The direct optimization of the design variable $\mathbf{D}$ in the end-user value $V(\mathbf{D})$ is a relatively simple and robust method but generates significant effort for assessing the value $V[i]$ in each iteration. A further disadvantage is that this black box model cannot explain why a design change increases or decreases the end-user value. There is a need for a gray box model with slightly increased complexity that explains the multi-variate function $f_{\mathrm{VD}}$ by additional variables and model parameters that are interpretable and helpful for the manufacturer of audio devices.

A good candidate for modeling the end-user value is the benefit-cost ratio (BCR), which can be written as:

$$
V(\mathbf{D})=\frac{f_{\mathrm{VP}}(\mathbf{P}(\mathbf{D}))}{\sum_{\forall j} C_{j}(\mathbf{D})}
$$

The function $f_{\mathrm{VP}}(\mathbf{P})$ in the numerator describes the benefit of the audio device based on performance features $\mathbf{P}=$ $\left\{p_{1}, \ldots, p_{k}, \ldots\right\}$. The sum of costs $C_{j}$ in the denominator represents the R\&D effort, manufacturing, after-sales service, field rejects, and other costs affecting the sales price. The features $\mathbf{P}$ and $\operatorname{cost} C_{j}$ are not independent from each other because both depend on the same design $\mathbf{D}$.

The features collected in $\mathbf{P}$ should cover all important aspects of the product in the multi-dimensional performance space. For the end user of audio products, the following dimensions are of particular interest:

- $\quad$ maximum output (max. SPL, power $P_{a, \max }$ )

- perceptual audio quality (spectral and spatial properties, distortion)

- efficiency (power consumption $P_{\mathrm{E}}$, heating, mobile operation time in battery-powered devices)

- reliability (probability of failure)

- endurance of external stress (overload, environment)

- size, volume, shape, weight

- convenience and ergonomics

- artistic product design

- technical story

- reputation of the brand, personal identification with the product

- enjoyment or hedonistic preference

The multivariable performance function $f_{\mathrm{VP}}(\mathbf{P})$ in Eq. (8) can also be approximated by a Taylor series by considering a small design change $\Delta \mathbf{D}$ and determining the variation in 
end-user value:

$$
\left.\Delta V \approx \lambda\left(\sum_{\forall k} w_{k} \Delta p_{k}(\Delta \mathbf{D})-\sum_{\forall j} \Delta C_{j}(\Delta \mathbf{D})\right)\right|_{\Delta \mathbf{D}<<\mathbf{D}}
$$

This linear approximation uses weights $w_{k}$, interpreted as a performance sensitivity, like the design sensitivity in Eq. (6) to scale the features $\Delta p_{k}$ and make performance comparable with the costs $\Delta C_{j}$. Both terms can be calibrated by the factor $\lambda$ to the metric used for assessing the end-user value. By choosing $\lambda=1$, the end-user value $V$ is expressed in monetary terms like the costs $C_{j}$, which simplifies the interpretation and psychometric evaluation of the value $V$.

Inserting the optimum design $\mathbf{D}^{*}$ determined by the iterative method in Eq. (7) gives the maximum value

$$
V\left(\mathbf{D}^{*}\right)=\frac{f\left(\mathbf{P}^{*}\left(\mathbf{D}^{*}\right)\right)}{\sum_{\forall j} C_{j}^{*}\left(\mathbf{D}^{*}\right)}
$$

with the optimum features $\mathbf{P}^{*}$ and costs $C_{j} *$. Contrary to the design sensitivity $s_{k}\left(\mathbf{D}^{*}\right)$ of the black box model in Eq. (5), the performance sensitivity defined as

$$
w_{k}[i] \approx \frac{\Delta V}{\lambda \Delta p_{k}}=\left.\frac{V[i]-V[i-1]}{\lambda\left(p_{k}[i]-p_{k}[i-1]\right)}\right|_{\Delta p_{j}=0 \forall j \neq k}
$$

usually becomes non-zero when converging to the optimum design $\mathbf{D}^{*}$. At this point the audio product cannot generate the ideal performance $\mathrm{p}_{\mathrm{k}}{ }^{\mathrm{i}}$ in all features but requires a compromise between features and cost. This compromise corresponds mathematically to a cancellation of the positive and negative contribution in the performance and cost in Eq. (9), generating zero design sensitivity $s_{k}\left(\mathbf{D}^{*}\right)$ at the optimum design $\mathbf{D}^{*}$. As a consequence, the optimum performance $\mathbf{P}^{*}$ is not identical to the ideal performance $\mathbf{P}^{\mathrm{i}}=$ $\left\{p_{1}{ }^{i}, \ldots, p_{k}{ }^{i}, \ldots\right\}$.

For example, a traditional home stereo loudspeaker with two 20-liter boxes designed 20 years ago might be closer to the ideal features in maximum output, flatness of the amplitude response, and distortion than a modern Smart Speaker in a 1-liter box. Today, the performance sensitivity $w_{V b}$ weighting the box size $V_{B}$ in Eq. (9) is significantly higher due to our modern life style. A reduction of the box volume $\Delta V_{B}$ compensates for decreased audio quality and increases the value of the product. The transparent gray box model allows us to directly compare this value increase $w_{V b} \Delta V_{B}$ with the contributions from other performance features, the cost structure, and the final sales price.

The performance sensitivity $w_{k}$ measured at the optimum design $\mathbf{D}^{*}$ can be used to calculate a tolerance $\Delta_{\mathrm{t}} p_{k}$ for each feature $\mathrm{p}_{\mathrm{k}}$ corresponding to a permissible relative loss $L_{v}$ (e.g., $L_{v}=10 \%$ ) in maximum end-user value:

$$
\Delta_{t} p_{k} \approx \frac{L_{v} V(\mathbf{D} *)}{\lambda w_{k}(\mathbf{D} *)}
$$

This tolerance is important for quality assurance and defining meaningful PASS/FAIL limits for end-of-line testing.

\section{PERFORMANCE MEASURES}

The evaluation of the performance $\mathbf{P}$ requires physical, psychological, and other non-technical measurement methods to generate numerical values on defined scales.

Standard measurements are available for evaluating the weight and volume of the product. Additional measures can easily be defined to describe flatness, compactness, and/or other geometrical properties.

The electro-acoustical efficiency expressed as the ratio of electrical input and acoustical output power can easily be measured for a single tone in the passband [3] and for broadband audio signals as well [4]. Although the sound pressure level SPL(r) at a point $\mathbf{r}$ and the total output power $P_{a}$ can be measured for an arbitrary stimulus, the rating of maximum acoustical output (maximum SPL and sound power $\left.P_{a, \max }\right)$ is more difficult and depends on the audio quality, reliability, and endurance required in the final application [5]. The following sections will focus on the physical and perceptual evaluation, which is a central topic in audio engineering. The influence of the measurement conditions will be discussed and characteristics with high performance sensitivity will be presented in three sets:

- $\mathbf{P}_{\mathrm{F}}$ for the final application condition (e.g., in a car)

- $\mathbf{P}_{\mathbf{S}}$ applied to standard condition (e.g., anechoic room)

- $\mathbf{P}_{\text {EoL }}$ used under manufacturing condition (e.g., test box)

The artistic design, brand, and other non-technical performance features might also become important in some consumer applications (e.g., headphones). Further research is required to find useful measures and psychological test and survey methods for assessing those non-technical performance features in audio engineering.

\section{PERFORMANCE IN THE FINAL APPLICATION}

The final application conditions have to be considered for defining the target performance and approving the final prototype. This evaluation uses measurements, models, and listening tests to generate physical and perceptual data that are useful for engineering and marketing as well. In order to generate meaningful results with minimum effort, it is useful to define test conditions that represent the typical usage of the device in the field and worst-case scenarios. Thus, relevant audio and test stimuli have to be selected, the position of device and the listening point or zone in the acoustical environment have to be determined, and listeners have to be qualified for rating the audio quality [7] and the end-user value $V$.

\subsection{Physical Evaluation}

It is convenient to measure the sound pressure at few points $\mathbf{r}_{1}$ in the sound field and generate meaningful physical characteristics describing the audio quality in the listening zone and evaluate the reliability and endurance (RE) of the product. Table 1 shows the estimated performance 
Table 1. Performance sensitivity (- no, * low, ** medium, *** high) of selected characteristics $\mathrm{P}_{\mathrm{F}}$ for assessing audio quality and reliability and endurance of the product in the final application.

\begin{tabular}{|c|c|c|}
\hline Characteristics $\mathbf{P}_{\mathrm{F}}$ & Audio Quality & Reliability, Endurance \\
\hline Complex transfer function $\mathrm{H}\left(\mathrm{f}, \mathbf{r}_{1}\right)^{1,2,3}$ & $* * *$ & $*$ \\
\hline Direct energy fraction (DEF) ${ }^{1,2,3}$ & $* *$ & - \\
\hline Amplitude compression ${ }^{1,2,3}$ & $* *$ & $* * *$ \\
\hline Harmonic distortion (THD, second, third-order) ${ }^{3}$ & $* *$ & $*$ \\
\hline Multi-tone distortion $(\mathrm{HD}+\mathrm{IMD})^{2}$ & $* *$ & * \\
\hline Modulated noise ${ }^{3}$ & $* *$ & * \\
\hline Incoherence $\mathrm{IC}(\mathrm{f}, \mathrm{n})^{2}$ & $* *$ & * \\
\hline Impulsive distortion (rub \& buzz) ${ }^{3}$ & $* * *$ & $* * *$ \\
\hline Nonlinear residuum $\mathrm{d}_{\mathrm{r}}(\mathrm{t})^{1}$ & $* * *$ & $* * *$ \\
\hline Perceptual sound attributes (MOVs) ${ }^{1}$ & $* * *$ & $*$ \\
\hline Perceptual overall audio quality ${ }^{1}$ & $* * *$ & * \\
\hline
\end{tabular}

${ }^{1}$ Assessed by using common audio stimuli.

${ }^{2}$ Measured with broadband test stimulus according IEC 60268-21 [5].

${ }^{3}$ Measured with sinusoidal test stimulus according IEC 60268-21 [5].

sensitivity for a list of highly independent characteristics $\mathbf{P}_{\mathrm{F}}$ that are selected and rated by the author to capture most of the important information in the final application.

\subsubsection{Testing With Artificial Stimuli}

Conventional measurement instruments prefer artificial test signals that allow fast measurements with sufficient signal-to-noise ratio, simplifying the evaluation of nonlinear distortion. The transfer function $H\left(f, \mathbf{r}_{l}\right)$ between input and output measured with a broadband signal (e.g., pink noise) has a high performance sensitivity for audio quality but less for RE. The direct energy fraction (DEF [8]) calculated from the $H\left(f, \mathbf{r}_{1}\right)$ considers the interaction between the loudspeaker directivity and the room properties generating the reflected sound. The DEF has zero performance sensitivity for RE because it provides almost no clues for any properties or defects. The variation of amplitude response $\left|H\left(f, \mathbf{r}_{1}, t\right)\right|$ versus time reveals the voice coil heating and other nonlinear compression effects. This characteristic is more sensitive for the RE than for audio quality.

A sinusoidal stimulus realized as a chirp or stepped sine wave is required to measure the total harmonic distortion (THD) and the second and third-order components. The room modes and sound reflections increase the complexity of the relative distortion responses. The interpretation can be simplified by presenting the absolute components in $\mathrm{dB}$ or referring the distortion components to the mean value of the fundamental response. Testing with a sparse multi-tone stimulus [5] reveals not only harmonics but also all kinds of intermodulation distortion generated in the full audio band.

A modulated noise measurement [9] using a sinusoidal test signal can detect turbulent air noise generated at a leakage in the dust cap or enclosure by the varying $\mathrm{AC}$ pressure. The incoherence [11] shows the energy part in the sound pressure output signal that is not correlated to the broadband white noise stimulus. This measure cannot distinguish between nonlinear distortion and additional noise that could corrupt the measurement [10].

All distortion metrics that calculate the power of the distortion components over a certain time interval (e.g., FFT) are less sensitive for impulsive distortion generated by loose particles and a rubbing coil. The impulsive distortion measurement according to IEC standard [5] exploits all symptoms of critical loudspeaker irregularities (rub \& buzz) and artifacts caused by digital signal processing (e.g., active protection systems) and has the highest performance sensitivity for both audio quality and RE.

\subsubsection{Testing With Audio Signals}

Since music and speech are usually preferred in listening tests, it is interesting to combine perceptual and physical evaluation for any audio input signal.

However, ordinary audio signals have non-stationary properties, meaning the spectrum and amplitude distribution may vary significantly over time. The correlation techniques that calculate the auto-spectrum and cross power spectrum require a stationary white noise signal to provide accurate results [12]. If the audio signal provides poor excitation of the loudspeaker at a particular instance, the complex transfer function becomes noisy and the incoherence becomes meaningless for describing the nonlinear distortion.

An interesting alternative is the adaptive linear modeling [10] of the device under test (DUT) as shown in Fig. 2. Both the DUT and linear filter are supplied with the same stimulus. The residuum is the difference $d_{r}=p$ - $p_{\text {lin }}$ between the linear model output with $p_{\text {lin }}$ and the measured sound pressure $p$, which is used for updating the filter parameters.

The parameter updating can cope with time varying properties such as voice coil heating and the changes in the visco-elastic suspension behavior caused by the nonstationary audio signal. The learning speed depends on spectral properties of the stimulus and stagnates if the input signal vanishes.

The residuum $d_{\mathrm{r}}$ contains noise and all kinds of distortion components generated by the transducer nonlinearities and undesired irregularities and defects (rub \& buzz). Signal analysis applied to the residuum $d_{\mathrm{r}}$ in the frequency and time domain (e.g., spectrogram) provides information that is comparable with standardized distortion metrics [10] measured with artificial test signals. 


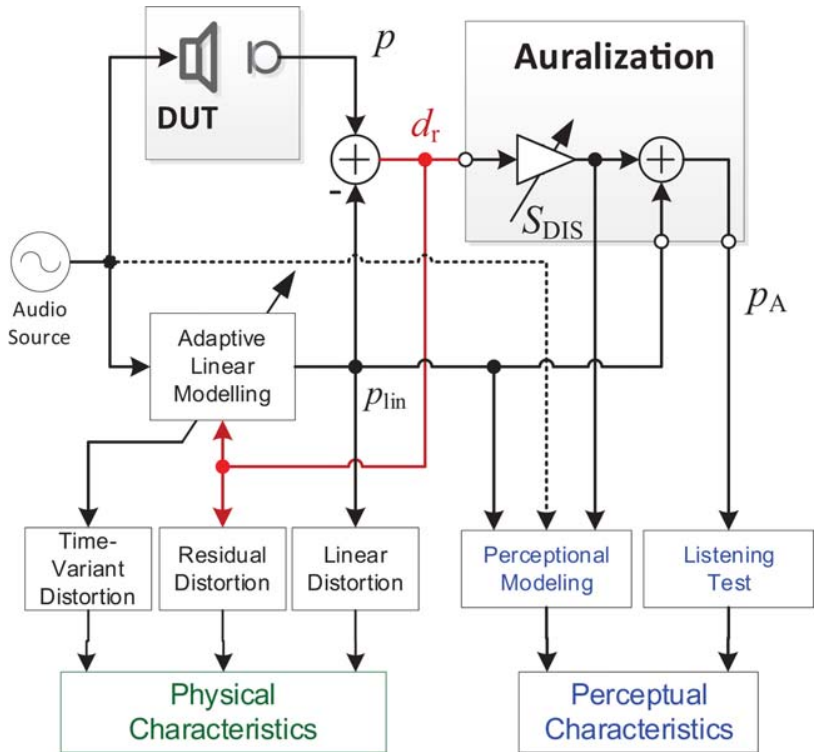

Fig. 2. Evaluation of the signal distortion in the final application by using ordinary audio signals.

The residuum $d_{\mathrm{r}}$ is the characteristic in Table 1 that has the highest performance sensitivity for both audio quality and RE-performance. Modern DSP algorithms for echo or active noise cancellation and beam forming generate an error signal that can be used for distortion analysis in the same way.

\subsection{Perceptual Evaluation}

An alternative approach to the physical measurement is the assessment of the listening impression of a typical or critical end user in the final application. Sound attributes such as clear, sharp, rough, and other verbal descriptions can be used to describe the product in all dimensions of the perceptual space [13].

Additional cognitive processing in our brain goes along with this and compares the sound attributes with some ideal conceptions [19]. The larger the distance between the real product and ideal point in the perceptual space, the lower the audio quality is rated by the listener. The conceptions of the ideal audio quality vary between the individual listeners but also over time due to experience, training, spirit of the age, and other cultural influences [7].

\subsubsection{Listening Tests}

Spontaneous listening without any systematic test strategy may be sufficient to detect severe design failures but is usually not sufficient to reliably rank high quality products. Listening tests need controlled condition (e.g., double-blind $\mathrm{AB}$ testing [13]) and statistical analysis to check the confidence of the test results.

Listening tests are time consuming and require restrictions in the number of test objects, audio samples, participants, and variations in the test condition (e.g., room). In practice, most of the listening tests are used for benchmark- ing a prototype with respect to other competitive products. However, those tests on existing products give very limited feedback about how the overall audio quality rating is related to physical and perceptual characteristics describing the different kinds of signal distortion.

The auralization technique is a simple and fast way to investigate the audibility of linear and nonlinear signal distortion and assess their impact on the overall audio quality [14]. This approach uses virtual test objects instead of the real competitive products, which are modifications of the same device under test, generated by attenuating or enhancing a particular distortion in the output signal.

A graphic equalizer can be used to compensate or synthesize peak and dips in the amplitude response. All-pass filters or time delay units can be used to modify the phase response to generate critical linear distortion in loudspeakers with multiple transducers or in multi-channel systems.

The auralization of the nonlinear distortion generated by a common audio signal can be realized by using the adaptive linear filter in Fig. 2. The residuum $d_{\mathrm{r}}$ comprising harmonic, intermodulation, and impulsive distortion as well as noise is weighted by the scaling factor $S_{\mathrm{DIS}}$ and added to the linear output $p_{\text {lin }}$ in order to generate an auralization output $p_{\mathrm{A}}$.

The auralization technique [14] can be used to generate a set of virtual test objects with varying distortion level (e.g., 3 - $\mathrm{dB}$ increment). This is a convenient basis to determine the audibility of the distortion by an automatic double-blind $\mathrm{AB}$ test using a forced step-down/step-up procedure [15]. The audibility threshold $T_{\mathrm{A}}$ corresponds to the scaling factor $S_{\text {DIS }}$ generating $75 \%$ correct answers. The set of virtual test objects can be used to rate the overall audio quality $P$ in a blind test and the end-user value $V$ in an open test with full information on the sales price and other product features. The variation of the end-user value $V$ versus $S_{\text {DIS }}$ reveals the performance sensitivity.

Fig. 3 illustrates the distortion auralization on three hypothetical examples each representing a typical woofer problem caused by nonlinear force factor $B l(x)$, stiffness $K_{M S}(x)$, and rub \& buzz. If the distortion is attenuated by $S_{\text {DIS }}=$ $-20 \mathrm{~dB}$, the rated audio quality $P \asymp P_{\text {lin }}$ and rated end-user value $V \asymp V_{\text {lin }}$ corresponds almost to a linear system generating no nonlinear distortion. At higher values of $S_{\mathrm{DIS}}$, the distortion becomes audible and reduces the audio quality $P / P_{\text {lin }}$. The relative decay of $V / V_{\text {lin }}$ is usually smaller due to the impact of other performance features (e.g., size).

The first woofer in Fig. 3 uses a cost-effective motor design with a nonlinear force factor $B l(x)$ generating amplitude modulation distortion over the full audio band [16]. The audibility threshold $T_{\mathrm{A}}=1 \mathrm{~dB}$ is close to the distortion level in the woofer $\left(S_{\mathrm{DIS}}=0\right)$. At this point the nonlinear distortion generates a significant decay in the audio quality and a high negative value of the performance sensitivity because amplitude modulation of the higher frequency components generates an unpleasant roughness sensation.

The second woofer uses a progressive suspension with nonlinear $K_{M S}(x)$ characteristic generating nonlinear distortion at low frequencies $(\mathrm{f}<200 \mathrm{~Hz})$ [16]. The distortion of this woofer is $10 \mathrm{~dB}$ above the audibility threshold $T_{\mathrm{A}}$ 

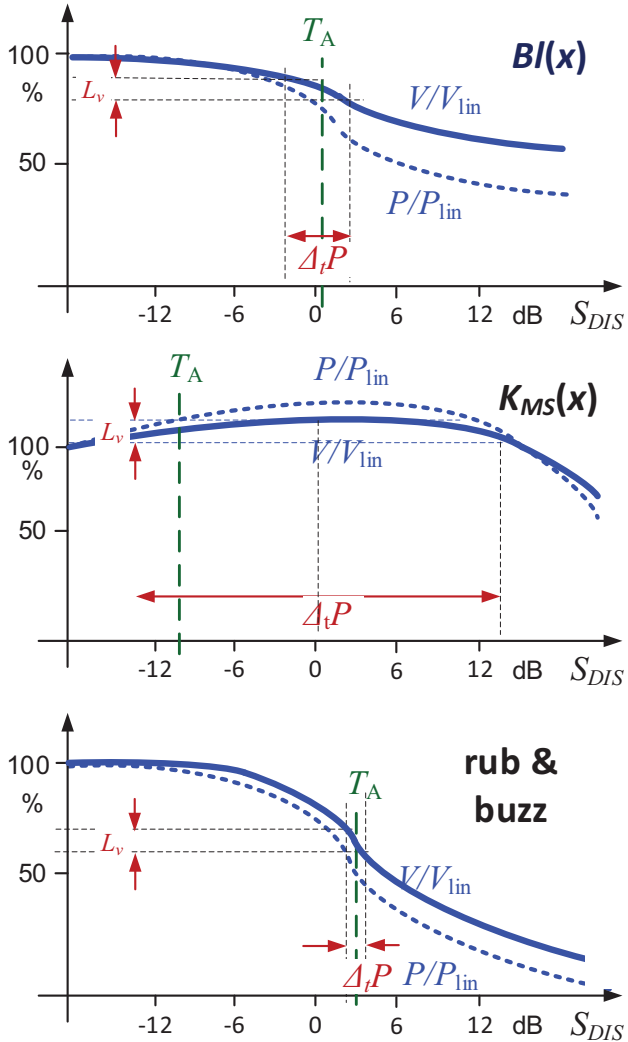

Fig. 3. Typical curve shapes of the rated end-user value $V / V_{\text {lin }}$ and audio quality performance $P / P_{\text {lin }}$ versus auralization gain $S_{\text {DIS }}$ in $\mathrm{dB}$ of the distortion generated by nonlinear force factor $B l(x)$, nonlinear suspension stiffness $K_{M S}(x)$, and impulsive distortion (rub \& buzz).

and evaluated as beneficial for the audio quality because the harmonic distortion improves the bass sensation. The performance sensitivity is positive for $S_{\mathrm{DIS}}<-6 \mathrm{~dB}$ and becomes negative at higher values of $S_{\text {DIS }}>12 \mathrm{~dB}$ because the higher dynamics of the harmonic components generate an aggressive impression that reduces the sound quality.

The third woofer has a rub \& buzz problem that occurs in the first prototypes and defective units failing the EoLtest. This problem usually generates a steep decay of the sound quality and a high negative value of the performance sensitivity at the audibility threshold $T_{\mathrm{A}}=3 \mathrm{~dB}$. Since this distortion usually contains high frequency components with an impulsive waveform [16], the properties are discordant to the musical content and become unpleasant and annoying for the listener if detected. The listener develops a rising awareness that makes the distortion unbearable to the listener. This sensitization is the opposite process of the adaptation [7] found in the perceptual and cognitive evaluation of loudspeaker and room interactions, which makes linear distortion in the amplitude response less critical after some time [17].

The most important results of the auralization technique are the audibility threshold $T_{\mathrm{A}}$ and the tolerances $\Delta_{t} p$ of the distortion level required to reduce the end-user value by a defined percentage ( $\left.L_{v}=10 \%\right)$ according to Eq. (10). The tolerances of the characteristics (e.g., THD) assessing the desired design properties (e.g., $B l(x)$ nonlinearity) are determined based on the performance sensitivity measured on the final prototype (using $S_{\mathrm{DIS}}=0 \mathrm{~dB}$ in the auralization). The tolerances for impulsive distortion required for assessing undesired loudspeaker irregularities and defects are calculated from the highest performance sensitivity, which is usually found at the hearing threshold $T_{\mathrm{A}}$ on defect units with rub and buzz problems. Those tolerances are important for the technical product specification and PASS/FAIL limits required in production.

\subsubsection{Perceptual Modeling}

A transformation of the physical metrics into perceptual and cognitive metrics removes information that is irrelevant for assessing the performance of the audio device as seen by the end user. The peripheral auditory system can be modeled as a bank of nonlinear bandpass filters with overlapping slopes that vary with the level of the input signal [18]. Those auditory filters generate an excitation pattern in which the energetic summation of the spectral components and other masking effects suppress smaller components and generate a smoothed output spectrum. This masking effect reduces the audibility of linear and nonlinear distortion. For example, a peak in the SPL response of a dense broadband audio stimulus generates a stronger impression of a discolored sound than a dip of the same size [7]. This sensation of discoloration [19] can be predicted by a model output variable (MOV) that assesses the mean deviation of the loudness spectrum of the audio signal $p(t)$ reproduced by the DUT and the original audio stimulus $u(t)$. Other MOVs using different weighting functions for the loudness deviation describe the sharpness [20] and the "bass enhancement" of the DUT [19].

The perception of the nonlinear distortion can be modeled by performing a separate auditory filtering of the residuum $d_{\mathrm{r}}$ and linear signal $p_{\text {lin }}$ using the adaptive linear filter in Fig. 2 and calculating the difference between the two excitation patterns in $\mathrm{dB}$. Considering the linear signal as a natural masker of the distortion, the maximum value of this difference found in the audio band is a simple MOV for describing the distortion-to-mask ratio (DMR) [19]. The higher this value, the more likely the distortion is audible. This MOV can explain the audibility of second and third-order harmonic distortion at a threshold of $0.3 \%$ at the optimum listening level of $60 \mathrm{~dB}$. The masking effect also explains the low audibility of the Doppler effect, which causes a frequency modulation of a high frequency signal (e.g., voice) by a low frequency signal (e.g., bass) [20].

However, the force factor $B l(x)$, inductance $L(x)$, and other transducer nonlinearities generate amplitude modulation distortion with a similar power spectrum but a modulated envelope in the time domain [16]. This temporal amplitude variation generates roughness in the perceived sound that makes the AM distortion $20 \mathrm{~dB}$ more audible than FM distortion [20]. MOVs describing the roughness can be calculated based on the temporal variation of the excitation pattern [20]. 
Other MOVs have been proposed for describing the auditory spaciousness based on the separated direct sound and diffuse sound, known as the interaural cross correlation coefficient (IACC) [21]. Further research on psychoacoustical modeling is required in the future to cover all dimensions of the perceptual space. A comprehensive set of MOVs is the basis for predicting the overall audio quality based on a linear or polynomial regression using ideal points, weights, and other model parameters [19],[22],[23].

\section{PRODUCT DEVELOPMENT}

The product development searches for the optimum design $\mathbf{D}^{*}$ by using particular performance characteristics $\mathbf{P}_{\mathbf{s}}$ that are either measured under standard condition or calculated based on the geometry and material data. Table 2 shows the performance sensitivity of selected characteristics for assessing the audio quality and RE performance.

Some of the characteristics (e.g., THD) are also listed in Table 1 and allow the performance of the product under application and standard condition to be compared. A difference in the values of corresponding characteristics reflects the influence of the positioning, distance, and room properties. However, the same tolerances $\Delta_{t} p$ measured in $\mathrm{dB}$ in the final application can also be applied to the corresponding characteristics measured under standard condition.

R\&D uses electrical and mechanical sensors, scanning, and fitting techniques for identifying free model parameters with a high performance sensitivity. For example, microphone scanning of the near field combined with holographic postprocessing reveals the direct sound at all points in the 3D space outside the scanning area. The on-axis response and directivity index are important for audio quality but less sensitive for the RE requirements.

Other models use lumped and distributed parameters to represent the electrical, mechanical, acoustical, and thermal properties of the audio device. The linear lumped parameters (TS [3]) describe the fundamental piston mode at low frequencies. Laser scanning combined with modal analysis gives more information about the cone vibration and sound radiation at higher frequencies [24]. Rocking and other undesired modes can cause the voice coil to rub, generating impulsive distortion and degrading the REperformance [25]. The nonlinear and thermal parameters reveal the physical causes of nonlinear distortion (THD, IMD), DC-displacement, and amplitude compression that reduce the maximum output, audio quality, and RE performance at high amplitudes.

The coincidence of a high peak value and high crest factor of the impulsive distortion [5] is a reliable indicator for irregular vibration (rub \& buzz). Plotting the impulsive distortion versus voice coil displacement reveals the condition under which the irregular vibrations are generated and gives clues about potential root causes (e.g., bottoming) [27].

Destructive testing complements the measurements of parameters and symptoms and reveals the weak parts of the design and the limits of the safe working range. Manufacturers use the results of all those measurements, fur-

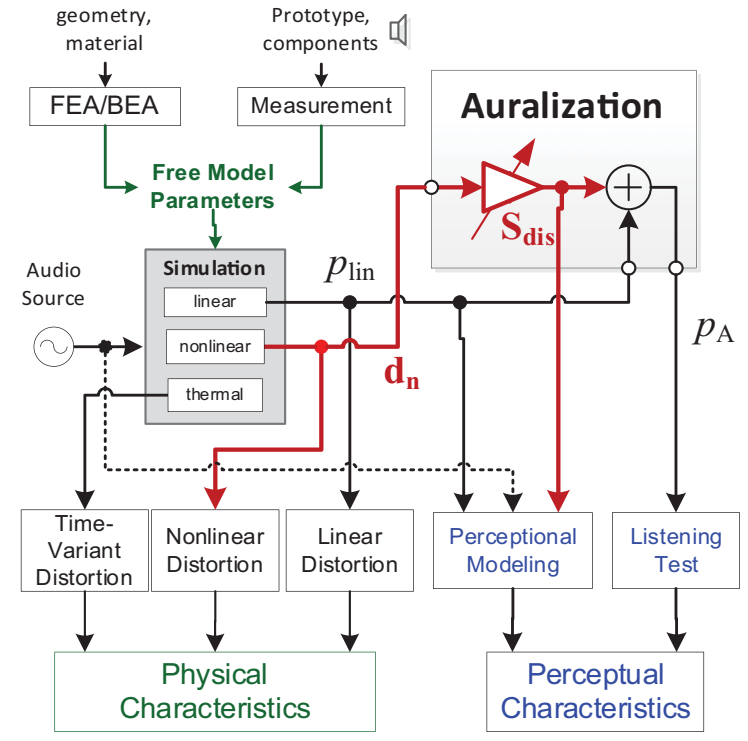

Fig. 4. Evaluation of the audio performance during product development based on numerical simulation and auralization of nonlinear signal distortion.

ther design details, and other performance characteristics to rate meaningful maximum values of voice coil displacement $X_{\mathrm{MAX}}$ [28], input voltage $U_{\mathrm{MAX}}$, and $S P L_{\mathrm{MAX}}$, which have a high performance sensitivity. The IEC standards [5] and [6] give the manufacturer sufficient freedom for this rating to generate maximum end-user value for a particular audio product in the final application. For example, the maximum voice coil displacement $X_{\mathrm{MAX}}$ is important for transducer and enclosure design and for adjusting active protection systems that prevent mechanical overload [29]. The maximum input voltage $U_{\mathrm{MAX}}$ and output characteristics $S P L_{\mathrm{MAX}}$ defined in IEC standard 60268-21 [5] are not only interesting marketing values but are required to test passive and active audio systems at a defined stimulus amplitude to measure the audio quality and other performance characteristics. However, this IEC standard [5] requires a $100 \mathrm{~h}$-long term test with a broad-band stimulus at input voltage $U_{\text {MAX }}$ to verify that the audio product can safely be operated at this level. For automotive, professional, and other applications it is required to perform accelerated life testing [30] and environmental testing [31] to verify the requirements in RE performance.

The free parameters of the lumped and block-oriented models used in electro-acoustics can be measured on available components and prototypes or can be calculated by numerical simulations as shown in Fig. 4. For example, finite element analysis (FEA) is a powerful tool for calculating electrical, mechanical, acoustical, and even thermal parameters for a chosen geometry and given material parameters. Boundary element analysis (BEA) is more beneficial for calculating the acoustical transfer functions between the velocity on the radiating surface and the sound pressure at any point in the sound field.

The electro-acoustical parameters are used in linear and nonlinear models to calculate internal state variables (e.g., 
Table 2. Performance sensitivity (- no, * low, ** medium, *** high) of selected performance characteristics $\mathrm{P}_{\mathrm{s}}$ evaluated under standard test conditions.

\begin{tabular}{|c|c|c|}
\hline Characteristics $\mathbf{P}_{\mathrm{s}}$ & Audio Quality & Reliability, Endurance \\
\hline On-axis fundamental transfer response ${ }^{3}$ & $* * *$ & $*$ \\
\hline 3D output, directivity ${ }^{3}$ & $* *$ & - \\
\hline Electrical impedance ${ }^{5}$ & $*$ & $*$ \\
\hline TS parameters ${ }^{5}$ & $* *$ & $*$ \\
\hline Modal parameters ${ }^{5}$ & $* *$ & $* *$ \\
\hline Nonlinear parameters ${ }^{4}$ & $* *$ & $* *$ \\
\hline Thermal parameters ${ }^{5}$ & $*$ & ** \\
\hline Amplitude compression ${ }^{3}$ & $* *$ & $* *$ \\
\hline DC-displacement $\mathrm{X}_{\mathrm{DC}}{ }^{5}$ & $* *$ & $* *$ \\
\hline Harmonic distortion (THD, second, third-order) ${ }^{3}$ & $* *$ & $*$ \\
\hline Multi-tone distortion (HD + IMD) ${ }^{3}$ & $* *$ & $*$ \\
\hline Distortion in reproduced audio signal [32] & $* *$ & - \\
\hline Modulated noise [9] & $* *$ & $*$ \\
\hline Impulsive distortion (rub \& buzz) ${ }^{3}$ & $* * *$ & $* * *$ \\
\hline Destructive test & $*$ & $* * *$ \\
\hline Maximum displacement $\mathrm{X}_{\mathrm{MAX}}{ }^{4}$ & $* * *$ & $* * *$ \\
\hline Maximum SPL ${ }_{\text {MAX }}$ or input voltage $\mathrm{U}_{\text {MAX }}{ }^{3}$ & $* * *$ & $* * *$ \\
\hline 100h-long-term test ${ }^{3}$ & $*$ & $* *$ \\
\hline Accelerated life test ${ }^{5}$ & $*$ & $* * *$ \\
\hline Environmental test ${ }^{5}$ & $*$ & $* * *$ \\
\hline
\end{tabular}

${ }^{3}$ Performed according IEC 60268-21 [5].

${ }^{4}$ Measured according IEC $62458[6]$.

${ }^{5}$ Measured according IEC 60268-22 CDV [4].

displacement), sound pressure output and nonlinear distortion components separated for each dominant nonlinearity (e.g., $B l(x), K_{M S}(x)$ ) in a vector $\mathbf{d}_{\mathbf{n}}$. Similar to the auralization based on the residuum in Fig. 2, the scaling of the nonlinear distortion vector $\mathbf{d}_{\mathbf{n}}$ with the weight $\mathbf{S}_{\text {DIS }}$ and the addition of the linear component $p_{\text {lin }}$ gives the auralization output $p_{\mathrm{A}}$ used for listening tests [32]. Since the linear and nonlinear modeling based on given parameters can be accomplished in real time on available PCs, the auralization of the dominant loudspeaker distortion can be performed for any audio stimulus.

This technique can be used to determine the audibility threshold $T_{\mathrm{A}}$ and performance sensitivity $w_{i}$ giving the permissible tolerances tolerance $\Delta_{t} p_{k}$ for the particular distortion. Without building a prototype, the combination of numerical simulation, auralization, and listening can decide whether the cost for a larger magnet size or shorting rings pay for the better audio quality provided.

The development is finished when an approved prototype or first pilot series is available that represents the optimum design $\mathbf{D}^{*}$, performance $\mathbf{P}^{*}$ with the permissible tolerances $\Delta \mathbf{P}^{*}$, and cost structure $\mathbf{C}^{*}$. This is the basis for finalizing the product specification and starting the manufacturing.

\section{MANUFACTURING}

The main target of manufacturing is to duplicate the prototype with the specified performance at minimum cost. From the perspective of quality assurance, the following objectives are important [33]:
- Automatic testing of $100 \%$ units at the end-of-line (EoL)

- Test smoothly integrated in production process (within given cycle time)

- Cost-effective and robust test hardware

- Simple use by operator (limit setting [34])

- Fast measurement and Pass/Fail classification (short stimulus, fast analysis [37])

- Reliable detection and separation of defective units

- Identification of the root cause of the defect

- Informative basis for an action plan (controlling assembling and supply chain) to maximize yield rate [36]

- Feedback to the product design (R\&D)

Those goals require modification of standard measurements:

- Near field measurements using a test box for shielding that are not comparable with final application and standard conditions [35]

- Transient measurement (no time for steady state condition)

- Minimum number of measurements providing results with low redundancy (no averaging)

- Large signal measurements (to activate nonlinearities and improve SNR)

- Provisions to cope with ambient production noise

- Properties of DUT changes over time (drying glue)

- Sensitive for symptoms of irregular behavior and defects 
- Considering the time-variant properties of adhesives in assembled transducers

Thus, the results measured at EoL are not directly comparable with the results measured in final application (FA) and under standard condition (R\&D).

\subsection{Sensitive Features for EoL-Testing}

There are two kinds of defects with different properties that require a different strategy in EoL testing [36]:

D1. Static defects with no significant change of the properties over the remaining product life.

D2. Dynamic defects with time-varying properties that degrade the perceptual and physical performance over time and potentially cause field rejects.

The static D1 defects already show all deficiencies at the end of the assembly line and can be detected based on PASS/FAIL limits derived from the product specification. Those PASS/FAIL limits are not adequate for the detection of dynamic D2 defects because the small symptoms found during EoL testing represent the initial phase of a destruction process but become worse over time in the final application [36]. Therefore, a trained operator or a sensitive test instrument is required to exploit early indications of dynamic D2 defects that are barely audible during testing but reveal problems in reliability and endurance. Some characteristics listed in Tables 1 and 2 show a high performance sensitivity for RE but have to be adjusted to the requirements in production.

Table 3 shows characteristics that can be measured in automatic tests considering the goals and restrictions at the EoL. The sinusoidal chirp with frequency dependent sweep speed and an amplitude profile optimally adjusted to the particular device [37] allows ultra-fast testing $(<1$ s). At least one microphone placed in the near field of the transducer is always required for assessing the acoustical output of any audio system.

The impulsive distortion measurement [5] and spectrogram $\operatorname{SPL}(f, t)[36]$ provide the highest performance sensitivity for abnormal vibration behavior (rub \& buzz) not intended by the design. The modulated noise measurement [9] is required to detect leakages in dust caps, cabinets, and irregular port noise. Sensing of the terminal voltage and input current is mandatory for identifying the lumped parameters of the manufactured transducer. The electrical input impedance, resonance frequency $f_{s}$, and quality factors $Q_{T S}, Q_{M S}, Q_{E S}$ are easy to measure but have low performance sensitivity for detecting dynamic defects in class D2. The absolute identification of the mechanical lumped parameters stiffness $K_{M S}$, force factor Bl, and moving mass $\mathrm{M}_{\mathrm{MS}}$ give more diagnostic information about the mechanical suspension, successful magnetization, and assembled components.

The fast measurement of the nonlinear parameters [39] allows the large signal performance to be evaluated, such as the maximum excursion, voice coil rest position, and

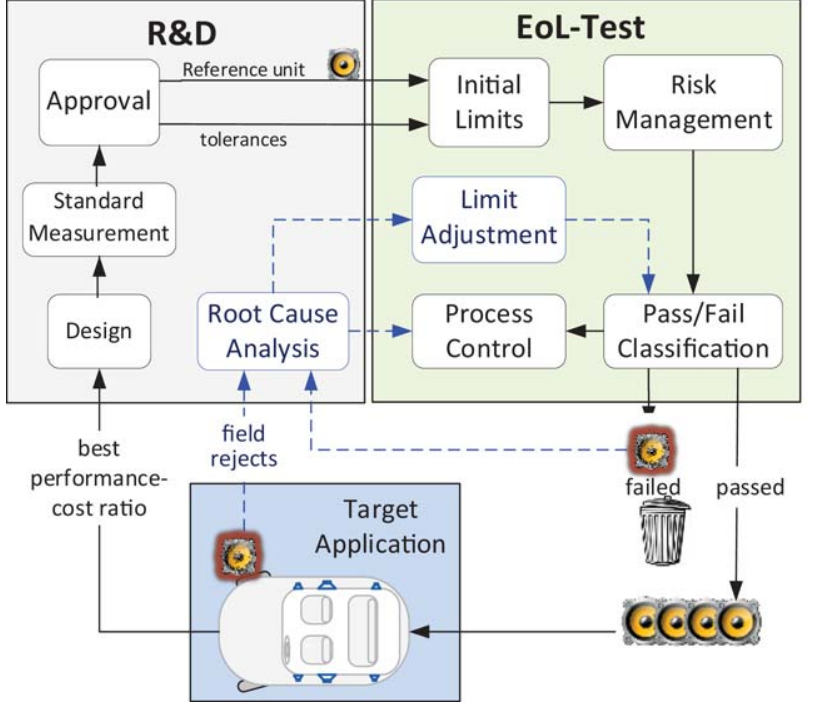

Fig. 5. Process of generating PASS/FAIL limits that will be later adjusted by the information generated by root cause analysis.

problems in the mechanical suspension at high excursions. Since the soft parts (spider, surround) cause the highest variability in the manufactured transducers, the characteristics describing the optimum working point of the voice coil in the mechanical system have a high-performance sensitivity with respect to reliability and endurance of the audio product.

\subsection{PASS/FAIL Classification}

Setting the PASS/FAIL limits is a cost-sensitive decision [36]. Tight limits reduce the likelihood of field rejects and the related after-sales $\operatorname{cost} C_{p}$ but increases the rejection rate and production cost $C_{E o L}$. Wide limits increase the yield rate but damage the reputation of the product in the market.

\subsubsection{Initial Limits}

Meaningful PASS/FAIL limits preventing static D1 defects can be generated if the technical product specification clearly defines the target performance $\mathbf{P}^{*}$ and the permissible tolerances $\Delta \mathbf{P}^{*}$, which should be the basis for an agreement between transducer supplier and system manufacturer.

Although some values in $\mathbf{P}_{\mathrm{S}}$ measured under standard condition are not directly comparable with the results in $\mathbf{P}_{\mathrm{EoL}}$ measured under EoL condition, it can be assumed that the agreed tolerances $\Delta \mathbf{P}$ expressed in $\mathrm{dB}$ are also valid in production. It is good practice to select a Golden Reference unit or an approved prototype representing the optimum performance $\mathbf{P}_{S}^{*}$ and to measure its performance $\mathbf{P}_{E o L}^{*}$ under EoL-condition as illustrated in Fig. 5. Then the upper limit, for example, can be calculated as $\mathbf{L}=\mathbf{P}_{E o L}^{*}+\Delta \mathbf{P}$ applying the tolerances $\Delta \mathbf{P}$ defined in specification [36]. 
Table 3. Performance sensitivity (- no, * low, ** medium, *** high) of performance characteristics $\mathrm{P}_{\mathrm{EoL}}$ measured in the EoL-test for detecting defects in performance and reliability.

\begin{tabular}{lll}
\hline \hline Characteristics $\mathbf{P}_{\mathrm{EoL}}$ & Audio Quality (D1, D2) & Reliability, Endurance (D2) \\
\hline Spectrogram SPL $(f, t)$ & $* * *$ & $* * *$ \\
Fundamental response (SPL) & $* *$ & $*$ \\
Averaged SPL response $^{3}$ & $* *$ & $*$ \\
Harmonic distortion (THD, second and third) & $* *$ \\
Impulsive distortion (e.g., rub and buzz) & $* *$ & $* * *$ \\
Modulated noise [9] & $* * *$ & $* *$ \\
Polarity & $* *$ & - \\
Electrical impedance & $* *$ & $*$ \\
Electrical parameters $\left(\mathrm{R}_{\mathrm{E}}, \mathrm{f}_{\mathrm{s}}, \mathrm{Q}_{\mathrm{TS}}, \mathrm{Q}_{\mathrm{MS}}, \mathrm{Q}_{\mathrm{ES}}\right)^{5}$ & $*$ & $*$ \\
Mechanical parameters $\left(\mathrm{M}_{\mathrm{MS}}, \mathrm{Bl}, \mathrm{K}_{\mathrm{MS}}\right)^{5}$ & $* *$ & $* *$ \\
Peak and bottom displacement & $* *$ & $* *$ \\
Maximum displacement limited by nonlinearities $\left(x_{B l}, x_{C}\right)^{4}$ & $* *$ & $* *$ \\
Stiffness asymmetry $\mathrm{A}_{\mathrm{K}}^{4}$ & $* *$ & $* * *$ \\
Offset $x_{\text {of }}$ in voice coil rest position & $* *$ & $* * *$ \\
\hline
\end{tabular}

${ }^{3}$ Performed according IEC 60268-21 [5].

${ }^{4}$ Measured according IEC $62458[6]$.

${ }^{5}$ Measured according IEC 60268-22 CDV [4].

\subsubsection{Risk Management}

The dynamic defects impairing robustness and reliability in class D2 need a different approach for determining the limit values $\mathbf{L}$. For many defects detected later in manufacturing and in the field, there is no reliable data available at the launch of a new type.

However, the mean value of properties $\overline{\mathbf{P}_{E o L}}$ and the standard variation $\sigma_{E o L}$ of the error free DUTs can be estimated based on statistical analysis applied to similar audio products [40] or easily determined by a pre-production run with a small number of DUTs assembled [41].

Those statistical parameters can be used to define, for example, upper limits as

$$
\mathbf{L}=\overline{\mathbf{P}_{E o L}}+z_{k} \sigma_{E o L}<\mathbf{P}_{E o L}^{*}+\Delta \mathbf{P}
$$

which are tighter than the initial limits defined by the technical specification in order to reduce the risk for field rejects caused by dynamic defects D2 [36].

The real quantile value $z_{k}$ is directly related via the quantile function to the probability for a false reject. Assuming a Gaussian distribution, a quantile value $z_{k}=3.29$ means that 1 false reject will be expected for 1,000 units tested. A larger real value of the quantile $z_{k}=3.89$ reduces the number of false rejects to 1 in 10,000 DUTs but increases the risk for a false acceptance causing a field reject. The optimal value of the quantile $z_{k}$ depends on the requirements in the particular application. A relatively small quantile $z_{k}$ will be used for premium audio products to trade a moderate increase of the EoL cost against a significant reduction of the risk for a field rejection. In a standard consumer product, a higher quantile $z_{k}$ will be used to reduce the cost of the product.

This approach has the advantage that the mean EoL cost $\bar{C}_{E O L}$ can already be estimated and used in the price calculation of the product.

\subsection{Learning From Field Rejections}

If the number of field rejects is higher than assumed in the risk management in Sec. 7.2.2, actions are required according to the Eight Disciplines (8Ds [42]) in problem solving.

An important step is the verification of the failure in the field reject by physical measurement, listening, and visual inspection. If the failure can be confirmed, an engineer tries to understand the different steps in the destructive process and identify the root cause. Traceability to metrics measured on the same DUT during the EoL-test shows the change of the properties over time and might reveal particularities of the DUT that are not found in other DUTs that passed the test. If those particularities also occurred in other field rejects, then this coincidence may be a good reason to adjust the PASS/FAIL limits in the on-going production as illustrated in Fig. 5. If a physical understanding of the root cause and the explanation of the destructive process is plausible, then the same remedies are applicable to similar products.

Generating an admissible hypothesis will be illustrated in a practical example:

A woofer rejected from the field generates no output. The visual inspection of the disassembled voice coil reveals a damaged coil. If the recorded EoL-test data applied to the same DUT also shows a voice coil offset and a significant increase of the stiffness asymmetry that do not appear on the other units, then the following casualty illustrated in Fig. 6 could explain the failure:

The surround part of the suspension system has been stored under wrong conditions, temporarily causing deformed corrugation rolls. After assembling the speaker, drying the adhesive, and applying a large stimulus to the transducer, the deformation of the surround will eventually disappear after break-in and cause an offset of the voice coil rest position and an increased asymmetry in the total stiffness curve $K_{m s}(x)$. In the field, the voice coil offset and the asymmetrical limiting of the surround will generate a 


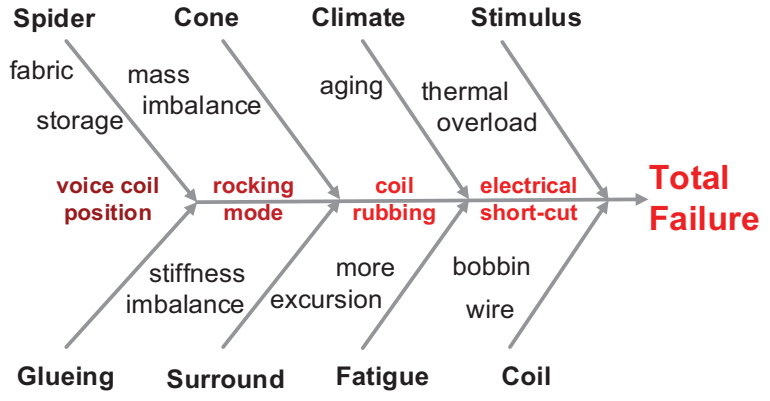

Fig. 6. Root cause analysis (fish-bone) of a total loudspeaker failure in the final application.

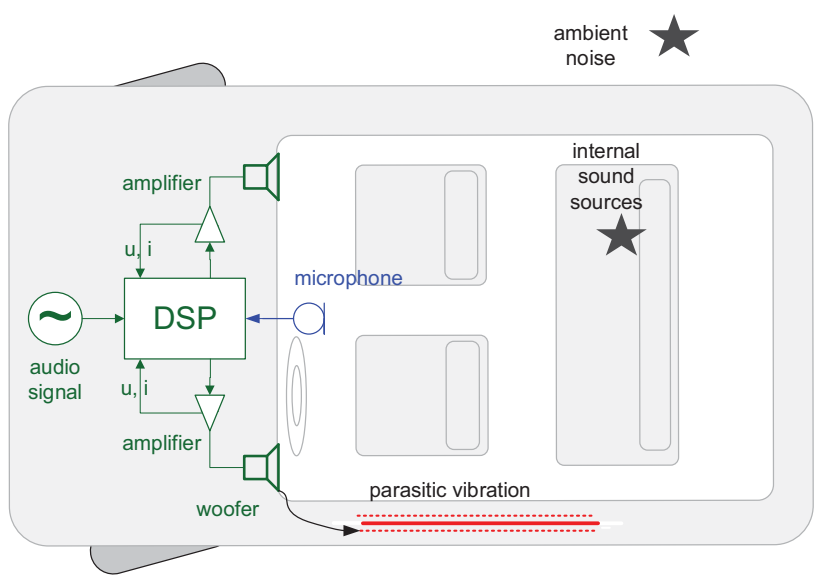

Fig. 7. In-situ testing in a car using available sensors and digital signal processing.

large restoring force, exciting a rocking mode due to an imbalance in the stiffness distribution over the circumference [25], [43]. The rocking mode causes a rubbing of the coil at the pole tips, damaging the wire insulation. Shorting the coil wire decreases the resistance, and the current causes a thermal overload that separates the wire from the voice coil former. The loose wire will soon be damaged in the gap, opening the connection to the electric source.

After verifying those hypotheses on other field rejects, the manufacturer can collect valuable knowledge about the physical cause of defects in class D2 and select useful actions minimizing both production and after sales costs.

\section{INTEGRATED IN-SITU TESTING}

Modern audio devices (e.g., smart speaker) provide all hardware and software requirements (sensor, amplifiers, DSP) for testing and monitoring the functionality of each unit over the remaining life time in the final application.

For example, Fig. 7 shows a car audio system using a smart amplifier with current sensing and a microphone for communication, hands-free operation, active noise cancellation, or other modern technologies [44]. The DSP has access to all digital signals coming from the audio source and sensors and given to the loudspeakers. Only software is required to generate meaningful diagnostic information and action in safety relevant application like a pedestrian sound generator in electric cars. There are two test scenarios that are significantly different with respect to stimulus, duration, and handling by a human operator.

\subsection{Self-Testing}

The first scenario is very similar to the EoL-test in Sec. 7 and uses artificial stimuli to shorten the test time and perform a comprehensive measurement of all sensitive characteristics listed in Table 3. The first advantage is that this self-test can be performed at any time and any location (e.g., on a car assembling line) without requiring any additional hardware. This test requires full ambient noise immunity, realized by detecting and repeating corrupted parts of the measurements. There is a second advantage that the selftest is not limited to the audio components themselves but can also detect parasitic vibrations of other equipment both airborne and excited by the mechanical structure. This is very important in cars where the rattling of lost screws, broken snap-ins, and loose panels can degrade the sound quality significantly.

The self-test will possibly only be activated in production or in workshops during after sales service.

\subsection{On-Line Monitoring}

A permanent measurement based on any audio signal selected by the end user can be performed by adaptive modeling of the signal paths from terminal voltage $u(t)$ to the measured sound pressure signal $p(t)$ and the sensed input current $i(t)$. This measurement can be combined with the possibly already existing system identification required for echo cancellation, active noise cancellation, beam steering, and other 3D sound solutions. The electric signals measured at the transducer terminals are the basis for the adaptive nonlinear control that provides valuable diagnostic information [45]. Table 4 shows the characteristics derived from the adaptive modeling.

The residuum $d_{r}(t)$ of the acoustical modeling has the highest performance sensitivity for defects degrading audio quality and RE as discussed in Sec. 5.1.2. However, the residuum contains nonlinear distortion from all speakers used in the car, parasitic vibrations, and ambient noise. Searching for the highest correlation of the residuum $d_{r}(t)$ with the voice coil displacement $x(t)$ provided by adaptive, nonlinear speaker control [45] reveals a defective speaker generating impulsive distortion. The electrical measurement of the linear and nonlinear transducer parameters, which is completely immune against ambient noise and sound generated by the other speakers, shows the voice coil rest position, instantaneous displacement, and progress of fatigue in the suspension. Those characteristics have the highest performance sensitivity for detecting a loss in reliability. This information can be used to replace a loudspeaker used in the field before a total failure of the transducer occurs. In other consumer applications where a replacement of the transducer is not practical, the adaptive nonlinear control system can reduce the peak excursion, which 
Table 4. Performance sensitivity (* low, ** medium, *** high) of selected transducer characteristics measured on-line in the final application (in-situ) while reproducing any audio signals.

\begin{tabular}{lll}
\hline Characteristics $\mathbf{P}_{\mathrm{F}}$ & Audio Quality & Reliability, Endurance \\
\hline Transfer response $H\left(f, \mathbf{r}_{1}\right)^{6}$ & $*$ & $*$ \\
Residuum of linear modeling $d_{r}(t)^{6}$ & $* * *$ & $* * *$ \\
Electrical impedance & $*$ & $*$ \\
Lumped parameters & & $* *$ \\
(linear, nonlinear) & $* *$ & $* * *$ \\
Mechanical stiffness $K_{M S}(t, x)^{7}$ & $*$ & $* * *$ \\
Offset $x_{\text {off }}(t)$ in coil rest position & $*$ & \\
\hline
\end{tabular}

${ }^{6}$ Measured by adaptive linear modeling of the electro-acoustical transfer function using a microphone.

${ }^{7}$ Measured by adaptive, nonlinear control based on current monitoring [45].

reduces the stress in the suspension and improves the RE performance.

In any case, on-line monitoring provides valuable information for the transducer manufacturer to check and improve the reliability and endurance of the product.

\section{CONCLUSION}

Searching for a comprehensive set of performance characteristics and making them comparable with cost is the basis for maximizing the end-user value. The assessment of audio quality, reliability, and endurance is a critical process that requires perceptual evaluation, physical measurements, and modeling.

The performance sensitivity introduced in this paper describes the impact of the characteristics on the end-user value and makes the characteristics comparable among themselves and with cost. The performance sensitivity of the characteristics assessing audio quality can be determined by auralization techniques using ordinary music in the final application environment.

The performance sensitivity is required for evaluating design choices, selecting meaningful characteristics, and defining target values and tolerances in technical product specifications. The tolerances are essential for generating initial PASS/FAIL limits for static defects (class D1) found in EoL-testing.

Additional risk management is required for dynamic defects (class D2) that generate no audible symptoms during EoL-testing but degrade the audio quality and reliability over time. A minor increase of production cost generated by false rejects are traded for a lower after sales cost spent for field rejects that damage the reputation. Traceability of the manufactured units opens new opportunities for root cause analysis of defective units, which is required to pin-point actions in process control. The performance sensitivity is again an essential criterion for evaluating the particular defect and finding the best solution.

Self-testing and on-line monitoring simplify the production, testing, and after sales service in the active audio system by exploiting available sensors and DSP to provide unique information with high performance sensitivity. Bringing the measurement instrument into the audio product not only reduces cost but allows the end-user value to be maintained for the remaining product life.

\section{REFERENCES}

[1] W. Klippel, "Evaluation of Audio Performance Over Product Life," presented at the 142nd Convention of the Audio Engineering Soc 142nd Convention of the Audio Engineering Societyiety (2017 May), convention paper 9737.

[2] M. W. Lipsey, Design Sensitivity: Statistical Power for Experimental Research (Applied Social Research Methods) (Sage Publications, 1990).

[3] L. Beranek and T. Mellow, Acoustics: Sound Field and Transducers (Academic Press, 2012).

[4] IEC, "Sound System Equipment - Part 22: Electrical and Mechanical Measurements on Transducers," International Standard IEC 60268-22:2019 CDV.

[5] IEC, "Sound System Equipment - Part 21: Acoustical (Output Based) Measurements," International Standard 60268-21::2018.

[6] IEC, "Sound System Equipment - ElectroAcoustical Transducers - Measurement of Large Signal Parameters," International Standard 62458::2010.

[7] F. Toole, Sound Reproduction, The Acoustics and Psychoacoustics of Loudspeakers and Rooms, 3rd ed. (Routledge, New York).

[8] J. Thompson, B. Smith, A. Warner, and J. -M. Jot, "Direct-Diffuse Decomposition of Multichannel Signals Using a System of Pairwise Correlations," presented at the 133rd Convention of the Audio Engineering Societyiety (2012 Oct.), convention paper 8807.

[9] W. Klippel and R. Werner, "Measurement of Turbulent Air Noise Distortion in Loudspeaker Systems," $J$. Audio Eng. Soc., vol. 59, no. 7/8 (2011 Jul./Aug.).

[10] W. Klippel and S. Irrgang, "Audio System Evaluation With Music Signals," presented at the 2017 AES International Conference on Automotive Audio 2017 AES International Conference on Automotive Audio (2017 Aug.), conference paper P4-2.

[11] P. Brunet and S. Temme, "A New Method for Measuring Distortion Using a Multitone Stimulus and NonCoherence," presented at the 121st Convention of the Audio Engineering Society 121st Convention of the Audio Engineering Society (2006 Oct.), convention paper 6877.

[12] J.S. Bendat and A.G. Piersol, Random Data, 4th ed. (Wiley \& Sons, Inc., 2010). 
[13] F.E. Toole, "Subjective Measurements of Loudspeaker Sound Quality and Listener Performance," J. Audio Eng. Soc., vol. 33, no. 1/2, pp. 2-32 (1985).

[14] W. Klippel, "Auralization of Signal Distortion in Audio Systems-Part 1: Generic ModelingPart 1: Generic Modeling," presented at the 135th Convention of the Audio Engineering Society 135th Convention of the Audio Engineering Society (2013 Oct.), convention paper 8992.

[15] C. Kaernbach, "Simple Adaptive Testing With the Weighted Up-Down Method," Percept. Psychoac., vol. 49, no. 3, pp. 227-229 (1991).

[16] W. Klippel, "Loudspeaker Nonlinearities - Causes Parameters, Symptoms," J. Audio Eng. Soc., vol. 54, no. 10, pp. 907-939 (2006 Oct.).

[17] S. E. Olive, P. L. Schuck, S. L. Sally, and M. Bonneville, "The Variability of Loudspeaker Sound Quality Among Four Domestic-Sized Rooms," presented at the 99th Convention of the Audio Engineering Soc 99th Convention of the Audio Engineering Societyiety (1995 Oct.), convention paper 4092.

[18] B. Moore, Hearing (Academic Press, 1995).

[19] W. Klippel, "Multidimensional Relationship Between Subjective Listening Impression and Objective Loudspeaker Parameters," Acustica, vol. 70, pp. 45-54.

[20] E. Zwicker and H. Fastl, Psychoacoustics, Facts and Models (Springer, 2007).

[21] J. Hirst W. J. Davies, and P. Philipson, "The Adaptation of Concert Hall Measures of Spatial Impression to Reproduced Sound," presented at the 120th Convention of the Audio Engineering Society 120th Convention of the Audio Engineering Society (2006 May), convention paper 6732.

[22] ITU-R, Method for Objective Measurements of Perceived Audio Quality (PEAQ), Recommendation BS.1387.

[23] S. Temme, P. Brunet, and D. B. (Don) Keele Jr., "Practical Measurement of Loudspeaker Distortion Using a Simplified Auditory Perceptual Model," presented at the 127th Convention of the Audio Engineering Society 127th Convention of the Audio Engineering Society (2009 Oct.), convention paper 7905.

[24] W. Klippel and J. Schlechter, "Distributed Mechanical Parameters of Loudspeakers, Part 2: Diagnostics," J. Audio Eng. Soc., vol. 57, pp. 696-708 (2009 Sept.).

[25] W. Cardenas and W. Klippel, "Rocking Modes (Part 2: Diagnostics)," presented at the 140th Convention of the Audio Engineering Soc 140th Convention of the Audio Engineering Societyiety (2016 May), convention paper 9496.

[26] W. Klippel, "Nonlinear Modeling of the Heat Transfer in Loudspeakers," J. Audio Eng. Soc., vol. 52, no. 1/2, pp. 3-25 (2004).

[27] W. Klippel and U. Seidel, "Measurement of Impulsive Distortion, Rub and Buzz and Other Disturbances, " presented at the 114th Convention of the Audio Eng Soc. (2003), preprint 5734.

[28] W. Klippel, "Assessment of Voice Coil Peak Displacement Xmax," presented at the 112th Convention of the Audio Engineering Society (2002 Apr.), convention paper 5508.
[29] W. Klippel, "Mechanical Overload Protection of Loudspeaker Systems," J. Audio Eng. Soc., vol. 64, no. 10, pp. 771-783 (2016).

[30] W. Klippel, "Mechanical Fatigue and Load-Induced Aging of Loudspeaker Suspension," presented at the 131st Convention of the Audio Engineering Society 131st Convention of the Audio Engineering Society (2011 Oct.), convention paper 8747.

[31] S. W. Hutt, "Ambient Temperature Influences on OEM Automotive Loudspeakers," presented at the 112th Convention of the Audio Engineering Society 112th Convention of the Audio Engineering Society (2012 Apr.), convention paper 5507.

[32] W. Klippel, "Auralization of Signal Distortion in Audio Systems Part 2: Transducer Modeling," presented at the 136th Convention of the Audio Engineering Society 136th Convention of the Audio Engineering Society (2014 Apr.), convention paper 9055.

[33] W. Klippel, End-Of-Line Testing, Assembly Line Theory and Practice, W. Grzechca (Ed.) (InTech, 2011), DOI: $10.5772 / 21037$

[34] G. Schmidle, "A Systematic Approach to Measurement Limit Definitions in Loudspeaker Production," presented at the 131st Convention of the Audio Engineering Society 131st Convention of the Audio Engineering Society (2011 Oct.), convention paper 8472.

[35] D. S. Schroeder and E. M. Long, "Reliability in Production Testing of Loudspeaker Components and Systems," J. Audio Eng. Soc., vol. 18, pp. 133-139 (1970 Apr.).

[36] W. Klippel, "Minimizing Costs in Audio Devices Through Efficient End-of-Line Testing," presented at the 145th Convention of the Audio Engineering Society 145th Convention of the Audio Engineering Society (2018 Oct.), convention paper 10049.

[37] S. Irrgang and W. Klippel, "Fast and Sensitive Endof-Line Testing," presented at the 144th Convention of the Audio Engineering Society 144th Convention of the Audio Engineering Society (2018 May), convention paper 9927.

[38] S. Hutt and L. Fincham, "Loudspeaker Production Variance," presented at the 125th Convention of the Audio Engineering Society 125th Convention of the Audio Engineering Society (2008 Oct.), convention paper 7530.

[39] W. Klippel and J. Schlechter, "Fast Measurement of Motor Suspension Nonlinearities in Loudspeaker Manufacturing," J. Audio Eng. Soc., vol. 58, no. 3, pp. 115-125 (2009 Mar.).

[40] A. Goldberg, "Quantifying Consistency in Loudspeaker System Production," presented at the 142nd Convention of the Audio Engineering Society 142nd Convention of the Audio Engineering Society (2017 May), convention paper 9713.

[41] M. Costanza Bellini and A. Farina, "Loudspeaker Performance Variance Due to Components and Assembly Process," presented at the 142nd Convention of the Audio Engineering Society 142nd Convention of the Audio Engineering Society (2017 May), convention paper 9714.

[42] Marcia M. Weeden, Failure Mode and Effects Analysis (FMEAs) for Small Business Owners and Non- 
Engineers: Determining and Preventing What Can Go Wrong (1952), ISBN 0873899180 , CLC 921141300.

[43] A. Schwock, W. Cardenas, M. Cobianchi, and W. Klippel, "Root Cause Analysis of Rocking Modes in the Nonlinear Domain," presented at the 142nd Convention of the Audio Engineering Society 142nd Convention of the Audio Engineering Society (2017 May), convention paper 9696.

[44] S. Irrgang and W. Klippel, "Self-Testing of Car Audio Systems," presented at the 2019 AES Interna- tional Conference on Automotive Audio 2019 AES International Conference on Automotive Audio (2019 Sept.), conference paper 2.

[45] W. Klippel, "Green Speaker Design (Part 1: Optimal Use of System Resources)," presented at the 146th Convention of the Audio Engineering Society 146th Convention of the Audio Engineering Society (2019 Mar.), convention paper 10138.

\section{THE AUTHOR}

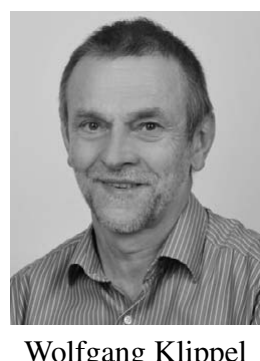

Wolfgang Klippel studied electrical engineering at the University of Technology in Dresden, in the former East Germany, where his initial studies focused on speech recognition. Afterward, he joined a loudspeaker company in the eastern part of Germany, where he was engaged in transducer modeling, acoustic measurement, and psychoacoustics. He later returned to his studies and received a Ph.D. in Technical Acoustics in 1987.

After spending a post-doctoral year at the Audio Research Group in Waterloo, Canada and working at Har-
man/JBL in Northridge, CA, he returned to Dresden in 1997 and founded Klippel $\mathrm{GmbH}$, a company that develops novel control and measurement systems dedicated to loudspeakers and other transducers.

Dr. Klippel has also been engaged as Professor of Electro-acoustics at the University of Technology in Dresden since 2007. His papers and tutorials on loudspeaker modeling and measurement-particularly those on large signal behavior and physical distortion mechanisms-are considered reference works in the field. 